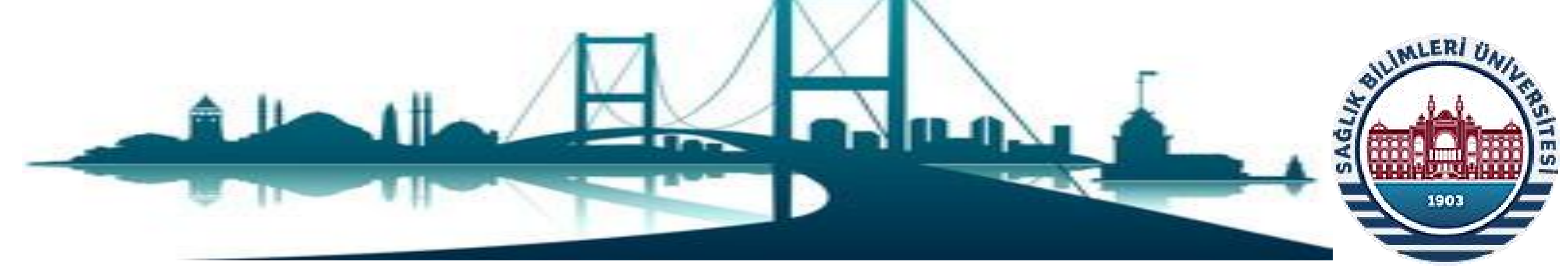

\title{
Comparison of the Baska mask, l-gel laryngeal mask airway and Classical laryngeal mask airway for effectiveness and complications in non-paralysed anaesthetised adult patients undergoing ambulatory surgery.
}

\author{
Isıker S., Goksu S., Bakan N. \\ Health Science University of Umraniye Training and Education Hospital \\ Istanbul, Turkey
}

Background: In this prospective randomised study, we compared the Baska mask with l-gel Laryngeal Mask Airway (LMA) and Classical LMA in patients undergoing general anesthesia for elective varicose vein operation.

Methods: After approval of local ethics committee and patient consent, 135 patients aged 18-64years of ASA group I-II were randomised into three groups as providing airway via;

\section{Baska Mask (Group I) \\ I-gel LMA (Group II) \\ Classic LMA (Group III).}

Same anaesthesia protocol was used (Induction; $1.5 \mu \mathrm{g} \mathrm{kg}-1 \mathrm{IV}$ fentanyl and $1-2 \mathrm{mg} \mathrm{kg}-1$ IV propofol, no muscle relaxant, maintanance; $1 \%-2 \%$ sevoflurane and $50 \%-50 \%$ mixture ofO2/air, $5 \mathrm{~L} \min -1)$.

The allocated LMA was inserted by the same anaesthetist when BIS was between $40 \%$ and $60 \%$.

Data recorded by unblinded observer as

-regarding number of attempts,

-ease of insertion (first time success rate, reposition, second attemps success rate

-insertion time,

-amount of leakage,

-oropharyngeal and systemic complications.

(Number Cruncher Statistical System 2007, One-way anova test, Tukey HSD, pearson chi-square test and Fisher-freeman-Halton tests, $p<0.05$ andp $<0.01-->$ statistically significant). -haemodynamic and respiratory parameters,

\section{Results and Discussion:}

The study conducted 128 patient $(49.2 \%$ males, $50.8 \%$ females and mean age of $41.34 \pm 11.28$ ).

-First time success rates of groups were 50, 81.8, 70.5 respectively. First time success rate of the group II was significantly higher $(p=0.038, p<0.05)$. Reposition rate and second attemps success rate were insignificantly lower in group II. So ease of insertion rate of the group II was higher than other groups $(p=0.038, p<0.05)$ (Table 1 ).

-Mean insertion time was significantly longer in the Group I as compared to Group II (25.73 \pm 6.94 vs. $21.98 \pm 4.88, p=0.018$; $\mathrm{p}<0.05$ ).

-Amount of leakage in the Group II was significantly lower than other groups $(p<0.01)$ (Figure 1$)$.

-All other parameters showed insignificant differences between the three divices.

Conclusion: Baska Mask, I-gel LMA and Classical LMA can be used in airway establishment during short surgeries but we suggest that; I-gel better than other two LMA as provides better seal with lower leakage, has short placement time and high ease of insertion rate.

Figure 1; Amount of leakage in $\mathrm{ml}$.

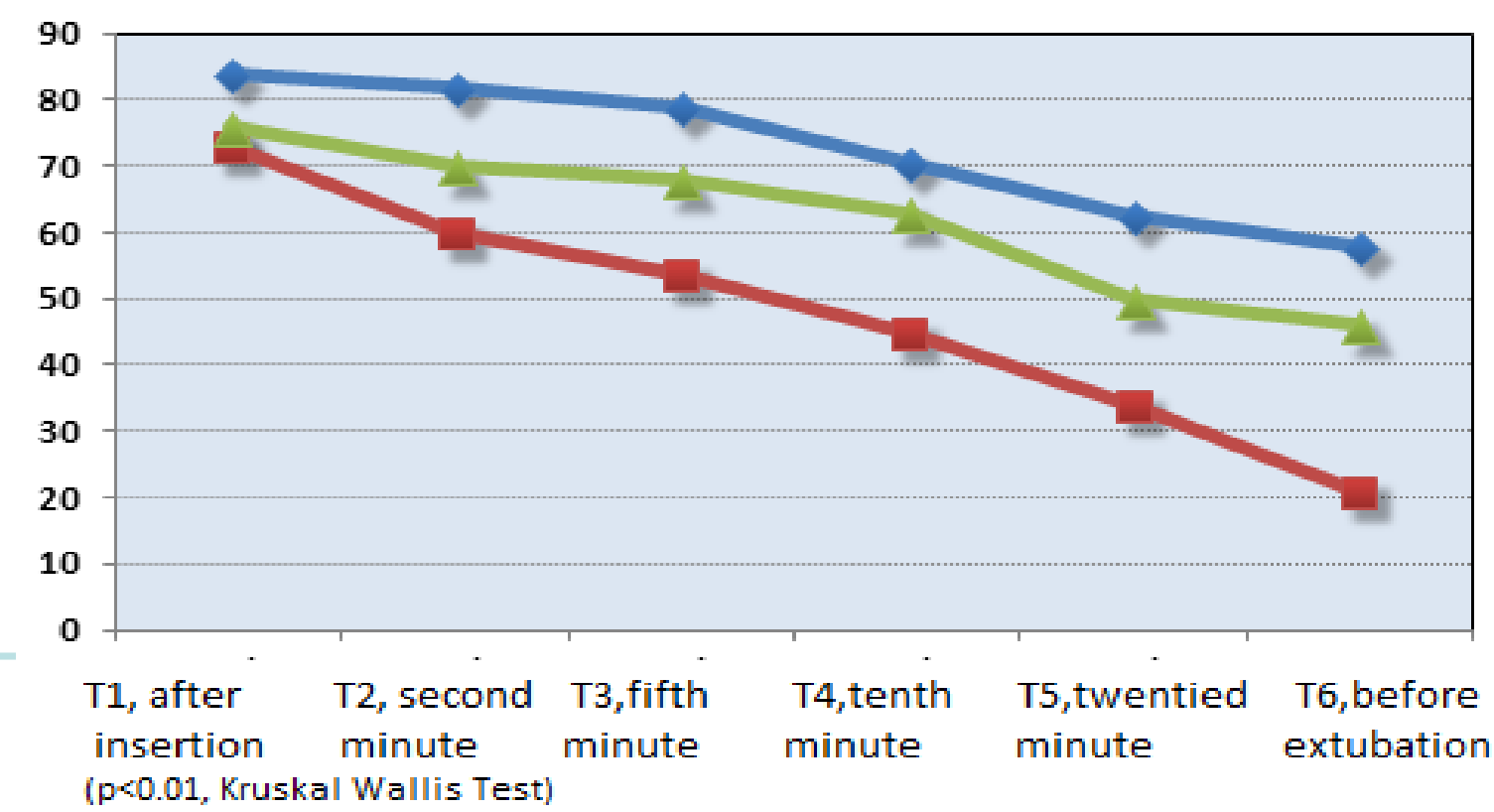

\begin{tabular}{|c|c|c|c|c|c|}
\hline \multicolumn{2}{|c|}{ Table 1; main data according to groups } & $\begin{array}{l}\text { Group I (Baska LMA, } \\
n=40) \\
n(\%)\end{array}$ & $\begin{array}{l}\text { Group II (l-gel , n= 44) } \\
n(\%)\end{array}$ & $\begin{array}{l}\text { Group III (Classic LMA } \\
, n=44) n(\%)\end{array}$ & $p$ \\
\hline \multirow{3}{*}{$\begin{array}{l}\text { Ease of insertion rate } \\
(p=0.038)\end{array}$} & First time success rate & $20(50.0)$ & $36(81.8)$ & $31(70.5)$ & $0,038^{*}$ \\
\hline & Reposition rate & $11(27.5)$ & $5(11.4)$ & $8(18.2)$ & $0.155^{*}$ \\
\hline & Second attemp success rate & $69(22.5)$ & $3(6.8)$ & $5(11.4)$ & $0.096 *$ \\
\hline \multicolumn{2}{|l|}{ Mean insertion time } & $25,73 \pm 6,94$ & $21,98 \pm 4,88$ & $23,41 \pm 6,09$ & $0,0180 * *$ \\
\hline
\end{tabular}

